\title{
AERODYNAMIC PROPERTIES OF PARTICLES IN THE GRAVITATIONAL FLOW OF A CHUTED BULK MATERIAL
}

\section{I.N. LOGACHEV ${ }^{\mathbf{1}}$, K.I. LOGACHEV ${ }^{1}$, O.A. AVERKOVA ${ }^{1}$, V.A. UVAROV ${ }^{\mathbf{1}}$, A.K. LOGACHEV ${ }^{1}$}

\author{
${ }^{1}$ Belgorod State Technological University named after V.G. Shukhov \\ (BSTU named after V.G. Shukhov), \\ 308012 Belgorod, Russia, Kostukova str., 46 \\ kilogachev@mail.ru, web page: http://www.bstu.ru
}

Key words: Granular Materials, Bulk Material Transfer, Air Suction, Local Exhaust Ventilation.

\begin{abstract}
Chutes are a linking element of transportation lines used for transfers of reprocessed materials from one transporting group or equipment to another. The mode of the chuted material motion and the nature of the associated aerodynamic processes are determined by the aggregate physical and mechanical properties of the material being transferred and structural design of chutes. Structurally chutes are subdivided into prismatic, cylindrical and pyramid-shaped (bin) chutes by shape and into vertical, tip and kinked chutes by the bottom slope angle. The most common in practice are tip chutes of a prismatic or a pyramid shape. The purpose of this work was the study of particle movement of granular materials in the sloping chute. In the result of the research, we revealed the following.
\end{abstract}

\section{INTRODUCTION}

Chutes are a linking element of transportation lines used for transfers of reprocessed materials from one transporting group or equipment to another. Transfer groups can be technically divided into four groups (Fig.1): conveyer to conveyer loading of material; conveyer to equipment loading of material; equipment to conveyer loading of material; equipment to equipment loading of material.

In all cases the material being transferred is first supplied to the funnel adjacent to the process equipment or mounted at the belt conveyer pully, and then the material is chuted by gravity to the lower transport conveyer or to the process equipment.

The mode of the chuted material motion and the nature of the associated aerodynamic processes are determined by the aggregate physical and mechanical properties of the material being transferred and structural design of chutes [1].

Structurally chutes are subdivided into prismatic, cylindrical and pyramid-shaped (bin) chutes by shape and into vertical, tip and kinked chutes by the bottom slope angle.

The most common in practice are tip chutes of a prismatic or a pyramid shape.

\section{PECULIARITIES OF A BULK MATERIAL MOTION IN CHUTES}

A granular material model selected to be used in study of the mechanical properties is a 
flow of crushed granite $\left(\rho_{1}=2750 \mathrm{~kg} / \mathrm{m}^{3}\right)$ which particles being $1.25-2.5 \mathrm{~mm}\left(d_{3}=1.56 \mathrm{~mm}\right)$ and $0.625-1.25 \mathrm{~mm}\left(d_{3}=0.74 \mathrm{~mm}\right)$ in size are by their shape and aerodynamic properties close to granular materials widely used in the ore preparation industry (crushed iron ore, chalk-stone, agglomerate, fines of agglomerated iron ore concentrate, etc.)

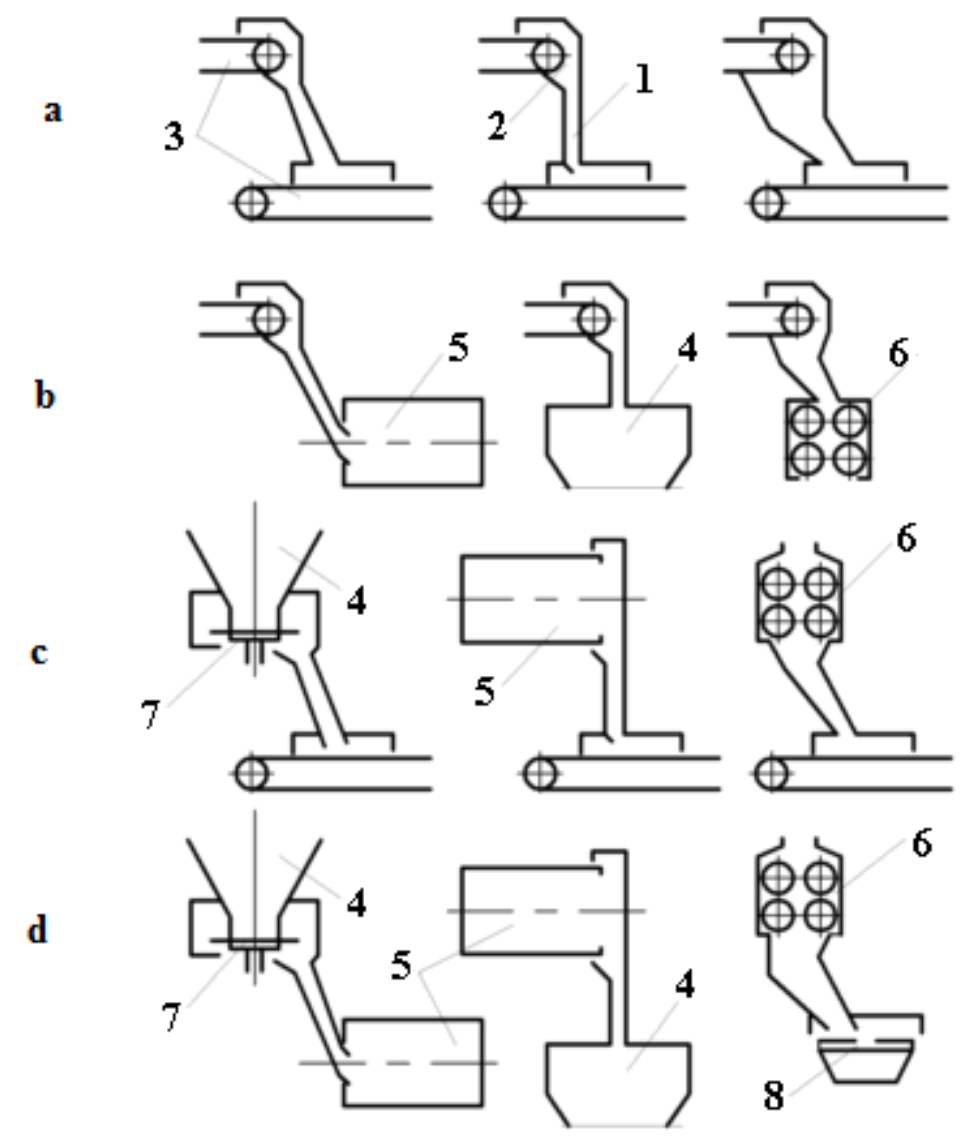

Figure 1: Conveyer to conveyer loading pattern (a), conveyer to equipment loading pattern (b), equipment to conveyer loading pattern (c) equipment to equipment loading pattern (d): 1 - chute; 2 - funnel; 3 - conveyers; 4 bins; 5 - drums (for the material cooling, mixing etc.); 6 - crushers; 7 - disc feeds; 8 - sieve

The study of modes of crushed granite particles motion and distribution in the tip chute cross-section as well as the measurement of the particles stream velocity was conducted on a test bench the main element of which was $3 \mathrm{~m}$ long chute of rectangular cross-section installed at various angles to the horizontal plane. The material was supplied to the cute from the upper bin through pre-tared diaphragms (Fig. 2).

\subsection{Modes of Motion}

When chuting crushed granite as in the case of grain flows in inclined pipes which for the first time ever were thoroughly studied by P.N. Platonov [2], there were three modes of motion observed: constrained motion mode, intermediate mode and unconstrained motion mode. 
The first mode feature is that a material is moved as an indiscrete mass with no noticeable discontinuity of contact among the particles. There is no bulk concentration gradient. The intermediate mode features local discontinuities in the indiscrete mass of particles. The third mode features the total decomposition of the indiscrete mass into separate particles or jets divorced from each other.

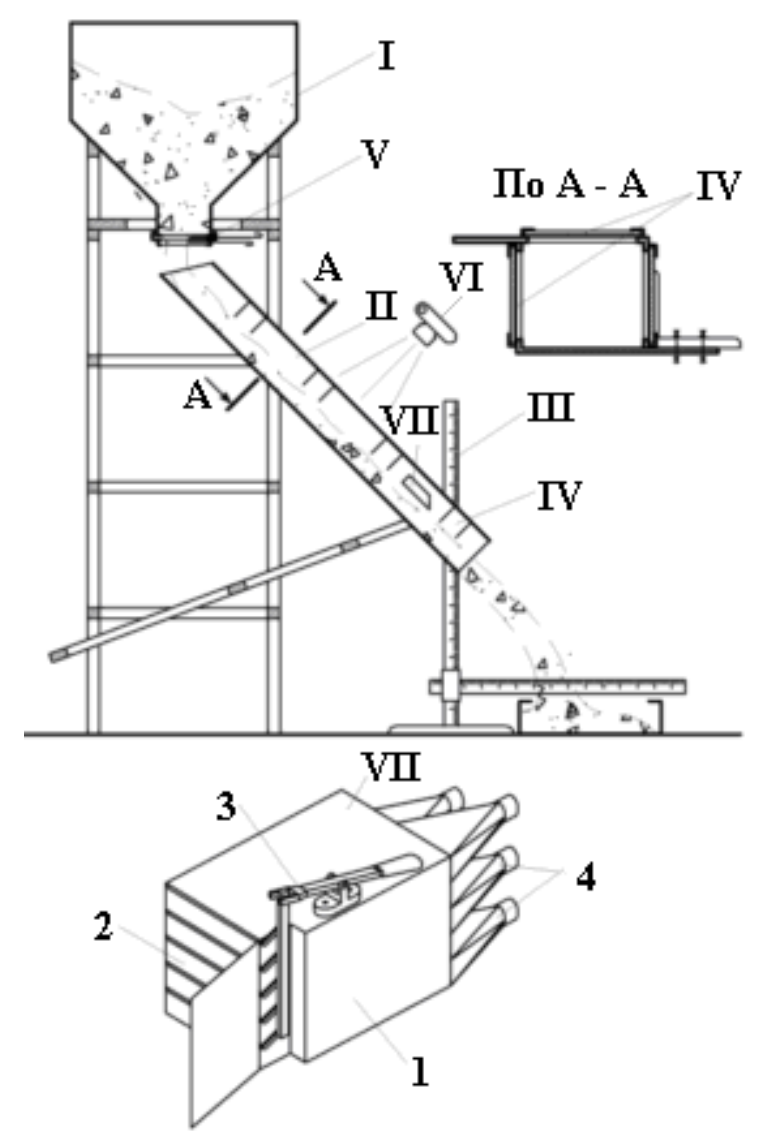

Figure 2: Diagram of the experimental arrangement for the study of physical and mechanical properties of a bulk material stream: I - upper bin; II - chute; III - coordinate spacer; IV - windows; V - diaphragm; VI - photo camera; VII - flow divider ( 1 - body, 2 - shelves, 3 - valve controller, 4 - bins)

Analyzing the motion of grain under high specific loads P.N. Platonov suggested using a chute inclination angle $\Theta$ as the parameter defining the nature of bulk material motion.

For instance, he noticed that the mode of constrained motion takes place if

$$
\Theta_{H}<\Theta<\Theta_{b},
$$

while the mode of unconstrained motion occurs when

$$
\Theta>\Theta_{6},
$$

where $\Theta_{H}, \Theta_{B}$ are external and internal friction angles respectively. 
When studying the stream of crushed granite particles in the tip chute we observed the mode of unconstrained motion at the chute inclination angles less than the internal friction angle. Therefore, an attempt was made to use the Froude number ${ }^{x}$ describing the stream kinetic movement instead of the chute inclination angle as a criterion for a change in the motion modes: $F r=g h / v_{1}^{2}$, where $h$ is a stream depth, m.

In order to clarify the physical meaning of this criterion for a bulk material motion in a chute under small specific loads we use the similarity of this motion with a motion of water in inclined drop structures. Let us evaluate the energy of particles in a cross section of the stream.

The flow strength of a material moving through surface $d s$ (Fig.3) per unit time with respect to datum 0-0 drawn through the lower point of the cross-section area in question is:

$$
d \ni=\beta_{1} \rho_{1} v_{1} \frac{v_{1}^{2}}{2} d S+\beta_{1} \rho_{1} v_{1} g d S \cdot y \cos \Theta .
$$

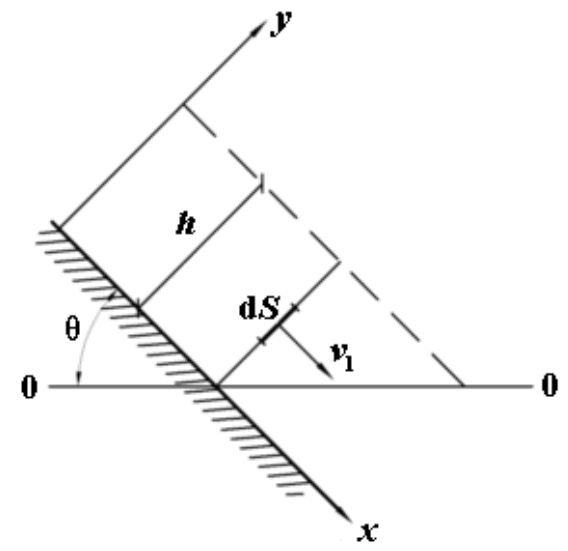

Figure 3: To the definition of a bulk material flow energy in a chute

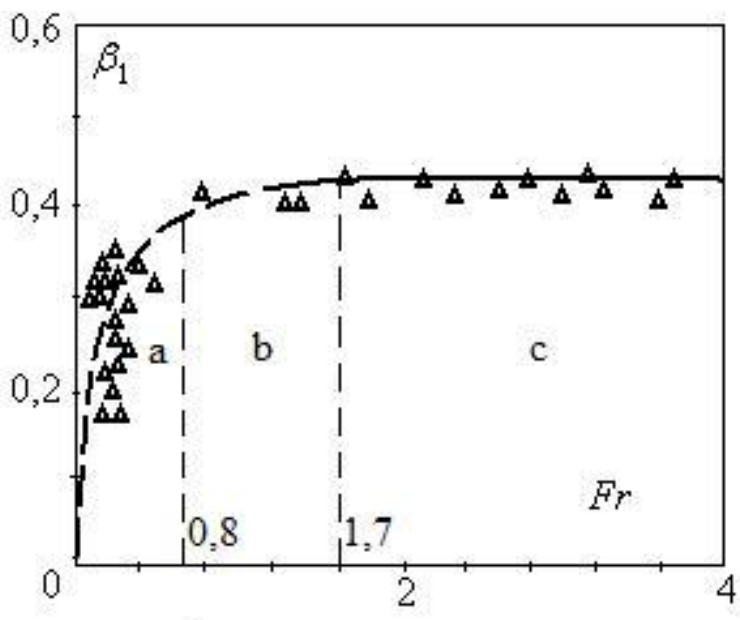

Figure 4: Dependence between a bulk concentration of crushed granite particles and Froude number

Then, the total material flow strength is

$$
\ni=\int_{S} \beta_{1} \rho_{1} v_{1} \frac{v_{1}^{2}}{2} d S+\int_{S} \beta_{1} \rho_{1} g v_{1} y \cos \Theta \cdot d S .
$$

Dividing the flow strength value by the bulk material weight flow rate

$$
G_{T}=\beta_{1} \rho_{1} g \bar{v}_{1} \cdot S .
$$

\footnotetext{
$x$ There is a good professional practice of applying this criterion in case with a bulk material flow with vertical chutes fully filled. For instance, when studying the motion of crushed graphite in a vertical pipe Z.R. Gorbis [4] defined the area of critical values of Froude numbers $1.65<F r_{c p}<5$ at which one mode is changed to another.
} 
and assuming that there is no bulk concentration gradient throughout the chute cross-section we obtain the energy per weight unit of the material passing through the cross-section area per time unit.

$$
\ni=\int_{S} v_{1}^{3} d S /\left(2 g S \bar{v}_{1}\right)+\left(\int_{S} y v_{1} d S\right) \cdot \cos \Theta /\left(S \bar{v}_{1}\right), .
$$

where $\bar{v}_{1}$ is a medium flow speed of the bulk material motion.

For a rectangular chute, when

$$
v_{1}=f(x, y), G_{T}=\beta_{1} g \rho_{1} B h \bar{v}_{1},
$$

we have:

$$
\ni=\frac{\alpha_{0} \bar{v}_{1}^{2}}{2 g}+\frac{k_{0} h}{2} \cos \Theta,
$$

where $\theta$ is the chute width, $\mathrm{m} ; h$ is a bulk material flow depth, $\mathrm{m} ; \alpha_{0}$ is the flow momentum correction factor equal to:

$$
\alpha_{0}=\left(8 \int_{0}^{h} v_{1}^{3} d h\right) /\left(s \bar{v}_{1}^{3} h\right)
$$

$k_{0}$ is the flow potential energy correction factor equal to:

$$
k_{0}=\left(8 \int_{0}^{h} v_{1} y d y\right) /\left(\bar{v}_{1} B \frac{h}{2}\right)
$$

It is obvious that in case with a gradientless motion of bulk material $\alpha_{0}=1$ and $k_{0}=1$.

The extreme value of specific energy $\ni$ in view of (7) occurs in our case when the flow depth $h=h_{\kappa p}$ :

$$
\frac{d \ni}{d h}=-\alpha_{0} G_{T}^{2} \cdot\left(\rho_{1} g \beta_{1} B h_{k p}\right)^{2} /\left(g h_{\kappa p}\right)+\frac{1}{2} k_{0} \cos \Theta=0
$$

or in the criterion form:

$$
F r_{\text {кp }}=\frac{2 \alpha_{0}}{k_{0} \cos \Theta},
$$

where $F r_{\mathrm{kp}}$ is the critical Froude number value equal to

$$
F r_{c p}=g h_{k p} / \bar{v}_{1}^{2} .
$$

When studying inclined drop structures [3] it was noticed that the critical Froude number value describes the transition of a subcritical fluid flow into a rapid flow. The latter is characterized by discontinuity of the fluid jet, especially at its free surface, and by an abundant aeration of the flow. The transition of the constrained motion of bulk material into the unconstrained motion is accompanied with a similar phenomenon: discontinuity of the jet and galloping motion of particles (saltation). Returning to condition (1) we can note that choosing $\mathrm{Fr}$ number as the transition criterion it is possible to consider the material flow rate in addition to the chute inclination angle. In other words, $\mathrm{Fr}$ criterion provides much information on a bulk material stream. 
Let us refer to the experiment. The research data concerning the nature of motion of a stream of crushed granite particles in a chute is shown on Fig. 2.4.

As in the case with the vertical motion of material [4], the dependence diagram $\beta_{1}=f(F r)$ is clearly divided into three areas that correspond to three modes of stream motion. In area $\mathrm{Fr}$ $>1.7$ (c), the bulk concentration is constant and virtually equal to the concentration of material at rest. This area corresponds to the mode of constrained motion. On the interval 0.8 $<\mathrm{Fr}<1.7$ (area (b)) the bulk concentration is sharply decreased, the material motion is characterized by local discontinuities among particle groups, and there is a velocity gradient in the flow depth. This area shall be conventionally called the transition area while the numbers within this area shall be the critical numbers. At Froude numbers below 0.8 (area $a$ ) dependence $\beta_{1}=f(\mathrm{Fr})$ is curvilinear and the lowest Froude number corresponds to the lowest bulk concentration. This area describes the mode of unconstrained motion. Note that according to equation (12) the area of the critical Froude numbers at inclination angles in question is

$$
2<F r_{c p} \leq 2,5,
$$

which is in line with the experimental data.

Various modes of bulk material motion complicate the analysis of the portrait of aerodynamic interaction of bombarding particles. Particles are flown around with air and the conditions of heat and mass exchange between the material and air change.

\subsection{Particle Distribution}

Distribution of particles over a chute cross-section in the mode of unconstrained motion is static. The theoretical prerequisites to the study of static regularities were made by L. Boltsman [5] who studied a stream of a large quantity of small elastic balls. He demonstrated that the particles concentration and speed are determined by the $F$ distribution function. This function pattern is defined by the differential equation:

$$
\frac{\partial F}{\partial t}+u_{x} \frac{\partial F}{\partial x}+u_{y} \frac{\partial F}{\partial y}+u_{z} \frac{\partial F}{\partial z}+X \frac{\partial F}{\partial u_{x}}+Y \frac{\partial F}{\partial u_{x}}+Z \frac{\partial F}{\partial z_{x}}=\Delta_{c} F
$$

where $X, Y, Z$ are external force components; $u_{x}, u_{y}, u_{z}$ are projections of particle velocities on coordinate axes; $\Delta_{c} F$ is the rate of change of the fixed point distribution function due to collision of particles.

However, the reduced equation in general defies solutions. There are approximate methods of solution known [6] with no regard to external forces that are determinative in our case. Therefore, in order to determine concentrations of particles we conducted experimental tests. There was a flow divider with five synchronized vales installed on the material motion path. Particles caught in the divider during a fixed interval of time were discharged from the divider bins and weighted. The experiments conducted in association with R.N. Shumilov [7] enabled to clarify the following pattern of motion of $0.625-1.25 \mathrm{~mm}$ crushed granite particles. A substantial part of particles is moving at the chute bottom. Moreover, the share of "bottom" particles is increased with the increase in the material flow rate (Fig. 5a) and with the decrease in the distance to the stream falling point at the chute bottom $(l)$. This is explained by a superposition of two processes occurring in a stream of airborne particles. The first is saltation process that is a galloping motion of particles resulting from the periodic impact of particles 
on the chute bottom, and the second process is the intercollision of particles.

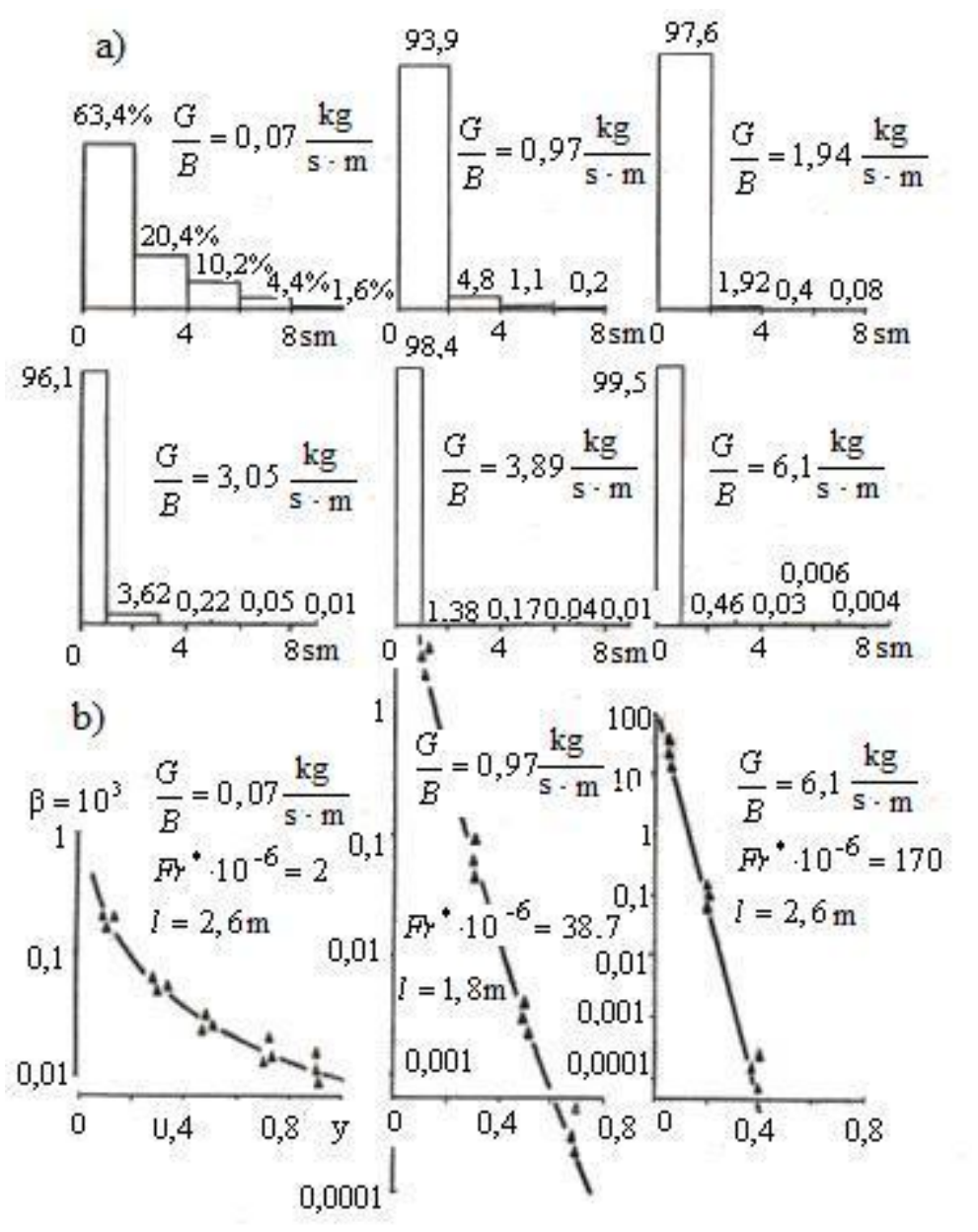

Figure 5: Distribution of crushed granite particles with the chute section height

At small flow rates or at a great distance $l$, when the concentration of particles is low, there is mostly a galloping motion with virtually no intercollision of particles. The transverse gradient of particles concentration is comparatively low.

At high flow rates, the concentration of particles reaches such values when intercollision of particles becomes so apparent that not every saltating particle having penetrated the thickness of particles flying above it is capable of leaving the stream. The quantity of particles drawn out from the stream and moving above it is small. The concentration gradient is high (Fig.5b)

The following quantitative characteristics were established. Distribution of particles with the channel height is clearly exponential

$$
\beta=\beta_{0} \exp \left(-a y^{n}\right),
$$

and the bulk concentration of particles at the chute bottom is subject to the flow continuity law $\beta_{0}=v_{1 \mu} \beta_{u} / v_{1}$, where $\beta_{\mu}$ is a bulk concentration of particles at the point of particles impact on the chute bottom; $v_{1 H}$ is the stream velocity at the chute inlet; $v_{1}$ is the stream velocity in 
the section in question. Coefficients $a$ and $n$ depend on the material flow rate and the distance to the section in question, i.e. $l$. The generalized parameter taken was a modified number that

$$
F r^{*}=\frac{G_{1} g}{v_{1}^{3} 6 \rho_{1}},
$$

(if $v_{1}=\bar{v}_{1}$ ) is related with the Froude number as follows:

$$
F r^{*}=\beta_{1} F_{r} .
$$

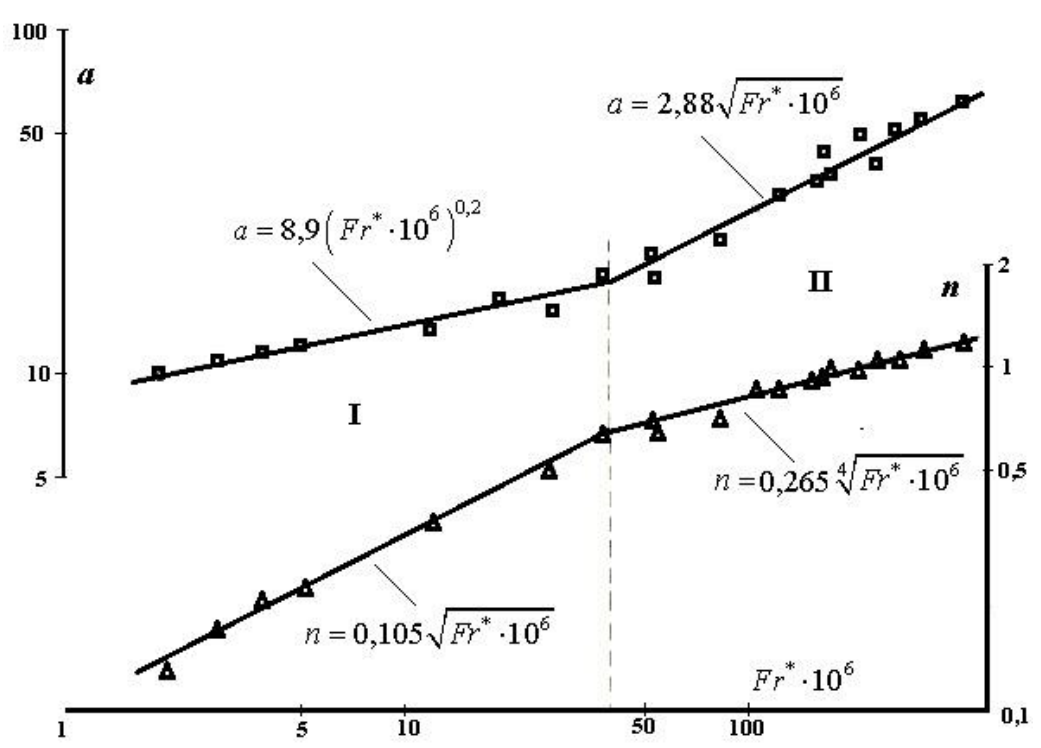

Figure 6: Variation of $a$ and $n$ coefficients with an increase in $\mathrm{Fr}^{*}$

The plots of $a$ and $n$ coefficients against $F_{r}^{*}$ are shown on Fig.6. Here, there are two areas clearly distinguished. The first area at $\mathrm{Fr}^{*} \cdot 10^{6}<40$ which we conventionally call the area of pseudo-uniform distribution of particles features a saltating motion and a comparatively low bulk concentration gradient $\left(n=0.1-0.67 ; n_{c p} \approx 0.3\right)$.

The second area, that is, the area of laminar motion, at $\mathrm{Fr}^{*} \cdot 10^{6}>40$ features a bed in which the most of colliding particles is moving and a small quantity of particles saltating above the bed. The concentration gradient is high $\left(n=0.67-1.2 ; n_{c p} \approx 1\right)$.

This peculiarity of the material motion in a tip chute makes the aerodynamic interaction pattern even more complicated and significantly changes the conditions of heat exchange.

\subsection{Motion Speed}

In industrial conditions a bulk material is typically chuted as a non-dense bed. Here, the effect of solid friction of particles is substituted with the effect of air drag forces, the frictional force resulting from the contact of particles with the chute walls and the gravity forces.

Unlike the gravity and air drag forces, the frictional force of particles contact is transient and very hard to determine. In order to obtain design data concerning the bulk material motion velocities we conducted experimental tests. There was the velocity of a stream of particles measured in various modes of motion in a tip chute of the experimental arrangement 
(Fig.2.). The velocity value was determined using two methods: the photographic method and the ballistic one. The first method consisted in measuring of a particle travel path in a time of the photographic shutter opening. Knowing the time of exposure $\Delta \tau$ and measuring the particles path sections obtained on photographic prints it is possible to determine the mean projection of particles velocity on the chute axis: $v_{1}=\frac{1}{N} \sum_{i=1}^{N} \frac{\Delta x_{i}}{\Delta \tau}$.

Section projections $\Delta x_{i}$ were determined using a ruler photographed together with airborne particles so that it was not necessary to consider the scale in photographing and photocopying. We used this method at low flow rates of bulk materials when the probability of superposition of paths could be neglected.

At low flow rates of a material the material velocity was determined using the second method which consisted in measuring the path of the material stream at the chute outlet. With the knowledge of the chute inclination angle and coordinates of the stream centerline, the material final velocity was calculated using a reduced dynamic equation of free settling particles. The centerline coordinates were determined using a coordinate spacer which horizontal axis was positioned in the material stream for more accurate measurements.

Solving the dynamic equation for a free-falling body

$$
\overrightarrow{\dot{v}_{1}}=\vec{g}
$$

in XOY coordinate system (Fig.7) with some transformations we obtain the following formula

$$
v_{\kappa}=\frac{x_{\kappa}}{\cos \Theta} \sqrt{\frac{g}{2\left(y_{k}-x_{k} \operatorname{tg} \Theta\right)}},
$$

which was used to calculate the velocity of particles at the chute outlet.

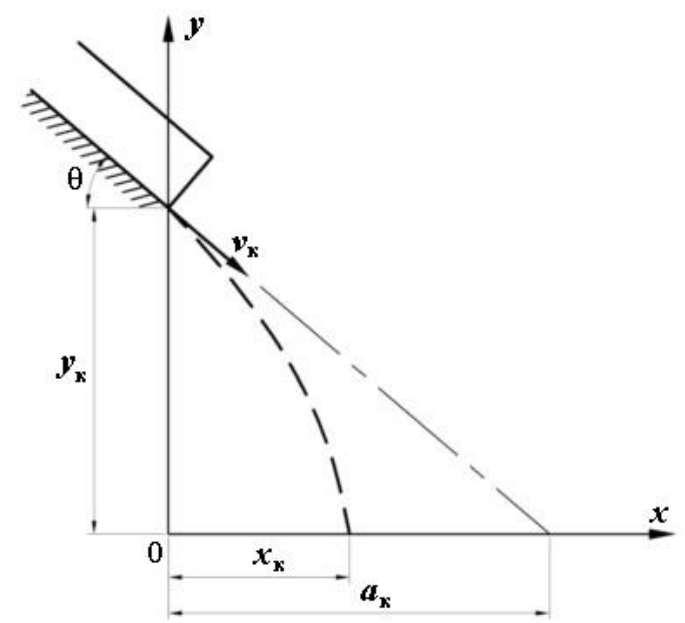

Figure7: To the definition of a path of bulk material particles poured from a tip chute

Since dynamic equation (18) does not account for the air drag forces relation (19) gives rather excessive results.

Experimental tests showed that a chuted stream of bulk material particles is virtually uniformly accelerated (Fig.8a).

The acceleration rate is: 


$$
a_{T}=g \sin \Theta \cdot\left(1-f_{m p} c \operatorname{tg} \Theta\right) .
$$

Conditional coefficient of the chute walls resistance to particle motion $f_{m p}$ depends on the modes of motion (Fig.8 b, c).

In case of the unconstrained motion this coefficient is lower than the coefficient of sliding friction $f_{c \kappa}$, and the relation $\delta=f_{m p} / f_{c \kappa}$ is: $\delta=0.5$ for the unconstrained motion, $\delta=1$ for the constrained motion.

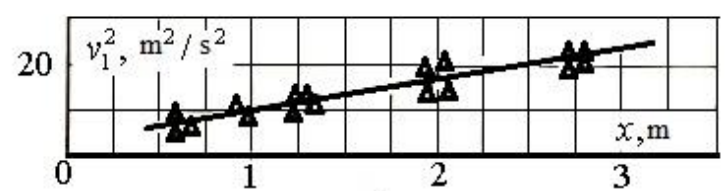

a)

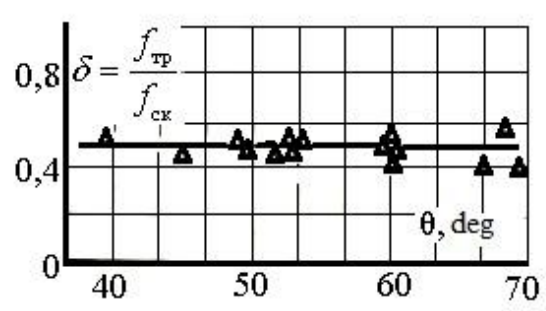

b)

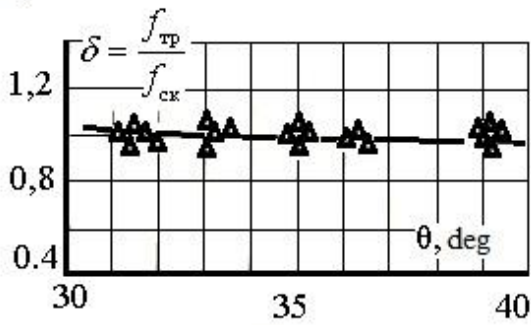

c)

Figure 8: Chute length variation in particle velocity (a) and the relation of the friction coefficient to the chute inclination angle in case of the unconstrained (b) and constrained (c) modes of bulk material motion

Considering the fact that $f_{c \kappa}$ varies over a wide range and depends on many factors (physical and mechanical properties of the transferred material, chute wall surface condition, etc.) practical calculations of local exhausts of transfer groups are recommended to be based on the assumption that $f_{m p}=0.5$.

Now, let us consider peculiarities of a material motion in kinked chutes. We will calculate the path and velocity of the conveyed bulk material stream (Fig. 9).

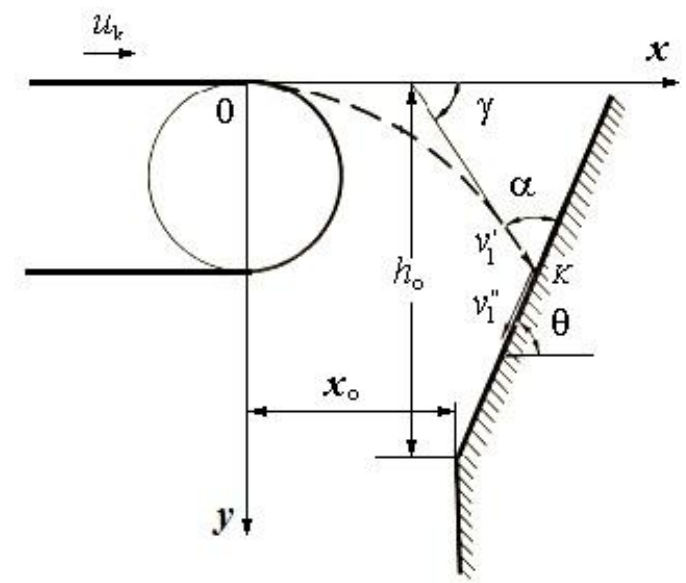

Figure 9: To the definition of the settling velocity of particles in the chute receiving funnel 
To this effect we use equation (18). By integrating this equation at the initial conditions $\left.v_{1}\right|_{t=0}=u_{\kappa},\left.x\right|_{t=0}=0,\left.y\right|_{t=0}=0$, we obtain:

a) for a jet trajectory (jet axis equation)

$$
x=u_{k} t, \quad y=g t^{2} / 2
$$

or

$$
y=0,5 g\left(x / u_{\kappa}\right)^{2}
$$

b) for velocity

or, in view of (22),

$$
v_{1}=\sqrt{u_{\kappa}^{2}+2 g y}
$$

$$
v_{1}=\sqrt{u_{\kappa}^{2}+\left(g x / u_{\kappa}\right)^{2}}
$$

where $u_{k}$ is the forward velocity of particles that is equal to the velocity of the horizontal conveyer belt, $\mathrm{m} / \mathrm{s}$.

Based on the chute wall position and the conveyer speed the discharged material flow may either contact the belt wall or not. The first case leads to a sharp change both in the jet trajectory and speed.

The flow-to-wall contact condition as is clear from relation (22) is determined by the following inequation:

$$
h_{0}>\left(x_{0} / u_{\kappa}\right)^{2} g / 2 .
$$

In order to find a point of contact ( $\mathrm{K}$ point having coordinates $\mathrm{x}_{\mathrm{K}}, \mathrm{y}_{\mathrm{K}}$ ) it is necessary to jointly solve path equation (22) and the obstacle surface equation. The latter in our case looks as follows:

$$
y=h_{0}-\left(x-x_{0}\right) \operatorname{tg} \Theta .
$$

Then

$$
x_{\kappa}=\frac{u_{\kappa}^{2}}{g}\left(\sqrt{1+2 g \frac{h_{0}+x_{0} \operatorname{tg} \Theta}{\left(u_{\kappa} \operatorname{tg} \Theta\right)^{2}}}-1\right) \operatorname{tg} \Theta \quad, \quad y_{\kappa}=\frac{g x_{\kappa}^{2}}{2 u_{\kappa}^{2}} .
$$

If the wall is vertical $(\Theta \rightarrow \pi / 2)$ it is easy to obtain

$$
x_{\kappa}=x_{0}, y_{\kappa}=g\left(x_{0} / u_{\kappa}^{2}\right) / 2 .
$$

As soon as the coordinates are available formula (24) can be used to determine the bulk material stream velocity at the moment of contact.

Elastic forces and the wall drag forces make the stream change its direction. An impact of a stream of irregularly-shaped particles is not an elastic impact in the strict sense, and, therefore, the angle of reflection is not equal to the angle of incidence for a stream in general or for single particles within the stream. R.L. Zenkov's studies [8] show that the angle of reflection for a bulk material stream is virtually equal to $\pi / 2$. The stream velocity after the impact is: $v_{1}^{\prime \prime}=K v_{1}^{\prime}$, where $v_{1}^{\prime}$ is the stream velocity at the wall contact, $\mathrm{m} / \mathrm{s} ; v_{1}^{\prime \prime}$ is the stream velocity after the wall contact, $\mathrm{m} / \mathrm{s} ; K$ is a correction factor accounting for the reduction in speed at the chute turn. 


\begin{tabular}{|c|c|c|c|c|c|c|c|c|}
\hline Chute turn $\alpha, \operatorname{deg}$ & 0 & 10 & 20 & 30 & 40 & 45 & 50 & 60 \\
\hline$K$ & 1.0 & 0.97 & 0.93 & 0.85 & 0.75 & 0.69 & 0.63 & 0.45 \\
\hline
\end{tabular}

In our case angle $\alpha$ is an acute angle between the tangent to the jet path in the contact point and the wall plane.

The tangent slope is determined after differentiating equation (22): $\operatorname{tg} \gamma=g x_{\kappa} / u_{\kappa}^{2}$,

Then it is obvious that $\alpha=180-\left(\Theta+\operatorname{arctg} \frac{g x_{\kappa}}{u_{\kappa}^{2}}\right)$.

The further calculation of the bulk material stream velocity is based on the formula: $v_{1}=\sqrt{2 a_{T} l+\left(v_{1}^{\prime \prime}\right)^{2}}$.

In case with significant drops of a fine material (when $h>0.5$ ) the medium drag force must be considered.

\section{CONCLUSIONS}

By its physical and mechanical properties, a bulk material stream in chutes features the unbound mode of accelerated motion with exponential distribution of particles by the channel cross-section depth which significantly changes the conditions for the dynamic intercomponent interaction, heat and mass exchange as compared to physically onedimensional models. By the particles distribution streams may be divided into two classes: streams featuring pseudo-uniform distribution of particles when a saltating motion of particles with a light concentration gradient prevails and streams featuring a laminar motion when the most portion of particles colliding each other is at the chute bottom and a small portion of saltating particles is above the layer of particles with a heavy concentration gradient.

The reported study was partly supported by RFBR, research project No. 14-4108005r_ofi_m.

\section{REFERENCES}

[1] Logachev, I.N. and K.I. Logachev. Industrial Air Quality And Ventilation: Controlling Dust Emissions. CRC Press, Boca Raton, (2014).

[2] Platonov, P.N.. Motion peculiarities of grain streams. Thesis ... Doctors of Engineering M (1969).

[3] Chertousov, M.L.. Hydraulics. M; L: Gosenergoizdat, (1962).

[4] Gorbis, Z.R. Heat exchange of dispersed through flows. M; L: Energiya, (1964).

[5] Boltsman, L. Gas theory readings. Gostekhizdat, (1956).

[6] Shidlovskiy, V.P. Introduction to rarefied gas dynamics. M: Nauka, (1965).

[7] Logachev, I.N. and Shumilov, R.N. Movement of bulk materials in chutes // Air ventilation and treatment: Collected papers of NIImetallurgventilyatsiya (1970). 4: 124129.

[8] Zenkov, R.L. Bulk cargo mechanics. M: Mashgiz, (1952). 